\title{
Self-interference Characterization for In-Band Full Duplex Underwater Acoustic Communications
}

\author{
Zheng Guo \\ Aijun Song \\ Department of ECE \\ The University of Alabama \\ Tuscaloosa, Alabama, USA \\ zguo18@crimson.ua.edu \\ song@eng.ua.edu
}

\author{
Mohammad Towliat \\ Leonard J. Cimini \\ Xiang-Gen Xia \\ Department of ECE \\ University of Delaware \\ Newark, Delaware, USA \\ \{mtowliat,cimini,xianggen\}@udel.edu
}

\author{
Chien-Chung Shen \\ Department of CIS \\ University of Delaware \\ Newark, Delaware, USA \\ cshen@udel.edu
}

\begin{abstract}
In-band full duplex (IBFD) communications attracts increasing attention in the underwater acoustic communication community due to its potential to increase spectral efficiency. The suppression of the self-interference remains the main obstacle to achieve IBFD in the ocean. Limited work in the literature has been done to characterize the self-interference in underwater acoustics. Here we analyze the characteristics of the self-interference based on the fieldwork, where self-interference measurements have been collected for multiple acoustic frequencies ranging from 20 to $180 \mathrm{kHz}$. The interference cancellation (IC) gain, as the performance metric of the digital self-interference suppression, is found to decrease when the acoustic carrier frequency increases. We propose to use the channel variation ratio (CVR) to characterize channel variability. Experimental results show that the CVR is larger at higher acoustic frequencies and high CVRs lead to the performance degradation in the self-interference suppression. Computer simulations have also been conducted to explain the experimental observations.
\end{abstract}

\section{KEYWORDS}

Self-interference characterization, in-band full duplex (IBFD), channel variation, underwater acoustic communications

\section{INTRODUCTION}

Underwater acoustic communications is important to marine exploration and discovery. The acoustic communication technology has experienced significant growth during the last few decades. A number of spectrally-efficient schemes, for example multipleinput/multiple-output systems and orthogonal frequency-division multiplexing, have been thoroughly investigated, leaving researchers willing to try more novel methods. In-band full duplex (IBFD) communications enables a pair of users to transmit to each other using the same frequency band at the same time. It may provide double spectral efficiency, increased throughput, and real-time feedback.

Permission to make digital or hard copies of all or part of this work for personal or classroom use is granted without fee provided that copies are not made or distributed for profit or commercial advantage and that copies bear this notice and the full citation on the first page. Copyrights for components of this work owned by others than ACM must be honored. Abstracting with credit is permitted. To copy otherwise, or republish, to post on servers or to redistribute to lists, requires prior specific permission and/or a fee. Request permissions from permissions@acm.org.

WUWNET'19, October 23-25, 2019, Atlanta, GA, USA

(C) 2019 Association for Computing Machinery.

ACM ISBN 978-1-4503-7740-9/19/10 . \$ \$15.00

https://doi.org/10.1145/3366486.3366510
Although IBFD has been shown feasible in radio communications, it has not been well explored for the underwater environment. It is recognized that the suppression of the self-interference in the underwater environment is the major obstacle to implement IBFD. This paper focuses on characterizing the self-interference and analyzing the performance of digital methods for the self-interference suppression.

Existing efforts in underwater IBFD have mainly focused on receiver designs and developing methods to cancel the local interference. Earlier work in [5] used frequency division to enable full-duplex acoustic communications. This scheme avoided the issue of cancelling the in-band self-interference and the limited acoustic frequency was not fully exploited. In [4], an asynchronous IBFD underwater acoustic communication system was developed. In the same effort [4], the nonlinear distortion caused by the power amplifier was considered. An over-parameterization-based recursive least squares algorithm was developed to suppress the nonlinear self-interference. In [7], a time-reversal method was proposed to separate the self-interference from the remote transmission. In [3], both digital and analog IC methods were examined for their effectiveness to suppress the self-interference.

Network protocols for IBFD acoustic networks have been considered. Using code division multiplex access techniques, the authors in [8] and [1] examined the feasibility of establishing full-duplex communications in underwater acoustic networks. A full-duplex based media access (MAC) protocol for ad-hoc underwater acoustic communication networks was developed in [9]. MAC protocols were developed for underwater acoustic IBFD networks to cope with different type of collisions in [6].

The characteristics of the self-interference, although important, have not been examined in detail in the literature. Due to the low propagation speed of sound and limited available bandwidth, underwater acoustic communications is very different from radio frequency communications. The unique propagation properties make the knowledge of the self-interference valuable and thus necessary. Channel variation is also a key factor limiting the performance of acoustic communications. Surface fluctuations, platform motion, and water volume dynamics are the causes for time-variation of the acoustic channel. But how does the channel variation impact self-interference suppression has not been studied in the literature.

IBFD requires co-located transmissions and receptions, within a short distance. The self-interference in IBFD is the result of the propagation in the near field, which is not well documented in 
the open literature. Understandings of the self-interference itself is the first step toward its cancellation. Therefore, our focus here, is to characterize the self-interference based on field measurements, over a range of acoustic frequencies from 20 to $180 \mathrm{kHz}$. Frequencydependency of the self-interference and interference cancellation (IC) gain are to be analyzed. The self-interference channel characteristics are to be presented to explain the IC gains observed in the field measurements.

The rest of the paper is organized as follows. Section 2 introduces the system model and the metric to measure the performance of the interference suppression, the IC gain. The IC gains across multiple acoustic frequencies are presented from the recent field measurements. Section 3 presents characteristics of the self-interference channel. Numeric simulations are also presented to explain the observed IC gains. Conclusions are given in section 4 .

\section{SELF-INTERFERENCE AND ITS CANCELLATION}

\subsection{Signal model}

IBFD underwater acoustic communications allows modems to exchange information using the same frequency band at the same time. While transmitting, the IBFD acoustic modem also receives the transmissions from another modem. Therefore, the self transmission is treated as interference to the remote signal that needs to be demodulated. To focus fully on the self-interference, we consider only the local interference without the remote signal. As illustrated in Fig. 1, the acoustic unit has a transmitter and a receiver, separated by some distance. Thus the baseband received signal at the local hydrophone is represented by

$$
r[n]=s[n] * h[n ; m]+w[n],
$$

where $s[n]$ denotes the transmitted symbols as the self-interference. $h[n ; m]$ represents the channel impulse response at arrival index of $m$ for the discrete time index of $n . w[n]$ is the Gaussian white noise, $w[n] \sim N\left(0, \sigma^{2}\right) . " * "$ denotes the convolution operation.

Assume the channel remains invariant during the observation window, Eq. 1 can then be rewritten into a vector-matrix form as

$$
r=S h+w
$$

where $\boldsymbol{r} \in \mathbb{C}^{M \times 1}$ and $\boldsymbol{r}=(r[n], r[n+1], \cdots, r[n+M-1])^{T}$, $\boldsymbol{h} \in \mathbb{C}^{N \times 1}$ and $\boldsymbol{h}=(h[n ; 0], h[n ; 1], \cdots, h[n ; N-1])^{T}, \boldsymbol{w} \in \mathbb{C}^{M \times 1}$ and $\boldsymbol{w}=(w[n], w[n+1], \cdots, w[n+M-1])^{T}, S \in \mathbb{C}^{M \times N}$ is the convolution matrix as in Eq. 3. $N$ is the length of the channel, $M$ is the length of observation window and $N \leq M$. We use $M=2 N$ throughout this paper.

$$
S=\left[\begin{array}{cccc}
s[n] & s[n-1] & \cdots & s[n-N+1] \\
s[n+1] & s[n] & \cdots & s[n-N+2] \\
\vdots & \vdots & \ddots & \vdots \\
s[n+M-1] & s[n+M-2] & \cdots & s[n+M-N]
\end{array}\right]
$$

The primary task that enables IBFD communication is the selfinterference suppression. To measure the performance of self-interference cancellation, we introduce the performance metric of IC gain.

\subsection{IC gain}

We only consider the digital cancellation to suppress the self-interference here. The digital cancellation method involves three steps: estimating the baseband self-interference channel, reconstructing the selfinterference signal, subtracting the reconstructed self-interference signal from the received signal. According to the signal model in Eq. 1, the signal after IC is given by

$$
r_{r e s}=\boldsymbol{r}-\hat{r},
$$

where $\hat{\boldsymbol{r}}$ is the reconstructed self-interference.

We define IC gain as the signal intensity ratio between before and after IC,

$$
G=10 \log _{10}\left(\frac{E\left[\boldsymbol{r}^{H} \boldsymbol{r}\right]}{E\left[\boldsymbol{r}_{\text {res }}^{H} \boldsymbol{r}_{\text {res }}\right]}\right),
$$

where $E[\cdot]$ denotes the expectation operation.

\subsection{IC gain at different signal frequencies}

An experiment was conducted to characterize the self-interference at multiple acoustic frequencies on February, 19, 2018 at the Black Warrior River, Tuscaloosa Alabama. The water depth at the site was 7.5 meters. The experiment configuration is shown in Fig. 1. The transducer was mounted 4 meters below the water surface. This configuration makes the reflection from surface and bottom arrive $5 \mathrm{~ms}$ and $4 \mathrm{~ms}$ later than the direct path. Corresponding channel structure can be verified in section 3.1. A high-precision hydrophone was mounted at the same transmission line, 1 meter below the transducer.

During the experiment, acoustic signals at nine frequencies were transmitted using three broadband omnidirectional transducers. The three transducers were mounted at the same depth, one at each time. The resonance frequencies of the three transducers are at 28 , 85 and $160 \mathrm{kHz}$, respectively. Two different linear power amplifiers were used. The 28 and $85 \mathrm{kHz}$ transducers used the same model of power amplifiers, but with different matching networks. Each transducer transmitted signals at three frequency points. The tested frequencies were 20, 25, 30, 75, 85, 95, 140, 160 and $180 \mathrm{kHz}$. The low-frequency transmissions at 20,25 , and $30 \mathrm{kHz}$ were sent out by the $28 \mathrm{kHz}$ transducer, with the source level around $170 \mathrm{~dB}$. The mid-frequency transmissions at 75,85 , and $95 \mathrm{kHz}$ were sent out by the $85 \mathrm{kHz}$ transducer, with the source level around $186 \mathrm{~dB}$. The high-frequency transmissions at 140,160, and $180 \mathrm{kHz}$ were sent out by the wideband $160 \mathrm{kHz}$ transducer, with the source level around $170 \mathrm{~dB}$. Nine m-sequence packets, each of which was one second long, were transmitted at the specified frequency. The symbol rate was set to be $5 \mathrm{kHz}$. The digital IC algorithm was applied to the field measurements. The least squares estimator was used to estimate the impulse responses. We computed corresponding IC gains according to Eq. 5. The IC gain results are shown in Fig. 2. The highest IC gain, measured, $39.2 \mathrm{~dB}$, was attained at the acoustic frequency of $20 \mathrm{kHz}$. The lowest IC gain, $20.6 \mathrm{~dB}$, was at the highest frequency of $180 \mathrm{kHz}$.

Note a high precision digital hydrophone was used in the field experiment. The received waveforms were sampled with 24-bit Sigma-Delta analog-to-digital converters (ADC), allowing a dynamic range of $132 \mathrm{~dB}$. The maximum achievable IC gain was far 


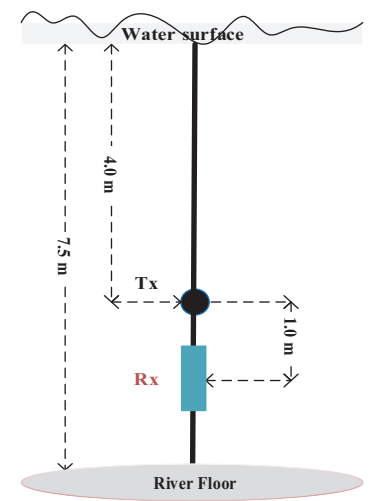

Figure 1: Experiment setup in the river experiment

smaller than this dynamic range. Thus the influence of ADC on digital cancellation was neglected.

It is clear in Fig. 2 that the IC gain decreased when the acoustic frequency increased over the frequency range of 20 to $180 \mathrm{kHz}$. The decrease in IC gain was about $19 \mathrm{~dB}$, which was significant, from the tested acoustic frequency range. The same trend existed within each of the three frequency clusters, where the same set of transmitter and receiver were used. The gradient of IC gain curve slowed down with the increased frequency. This change of gradient indicates the IC gain decreases faster at lower frequencies.

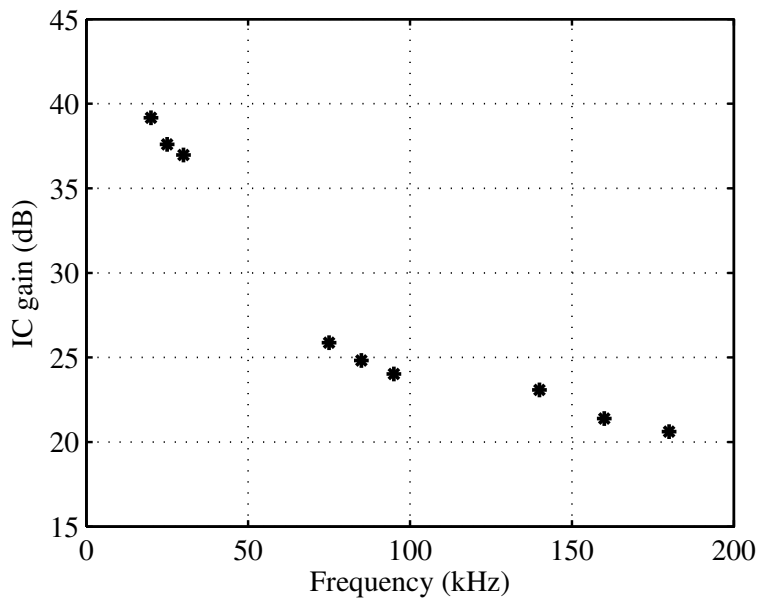

Figure 2: IC gain at nine acoustic frequencies, from 20 to 180 $\mathrm{kHz}$, based on the field measurements in a local river.

\section{SELF-INTERFERENCE CHANNEL CHARACTERIZATION}

To further understand the frequency-dependency of the IC gain achieved by the digital cancellation method, we focus on the characteristics of the self-interference channel in this section, specifically on channel coherence and channel variations.

\subsection{Measured channel impulse responses}

Using the experimental data, we extracted the measured impulse responses at multiple acoustic frequencies. Since the transducers had different transmitting voltage responses at different carrier frequencies, we normalized the measured channel impulse responses to a total energy of one.

The impulse responses are shown in Fig. 3 to Fig. 5 for three acoustic frequencies: 30, 95, and $180 \mathrm{kHz}$. The three impulse responses had a similar arrival structure. This was explained by the same transmitter-receiver geometry in the experiment for all the transmissions. The three impulse responses had strong, stable returns at the arrival-time of $5 \mathrm{~ms}$. After $5 \mathrm{~ms}$, the arrivals at $30 \mathrm{kHz}$ were more stable than those at 95 and $180 \mathrm{kHz}$.

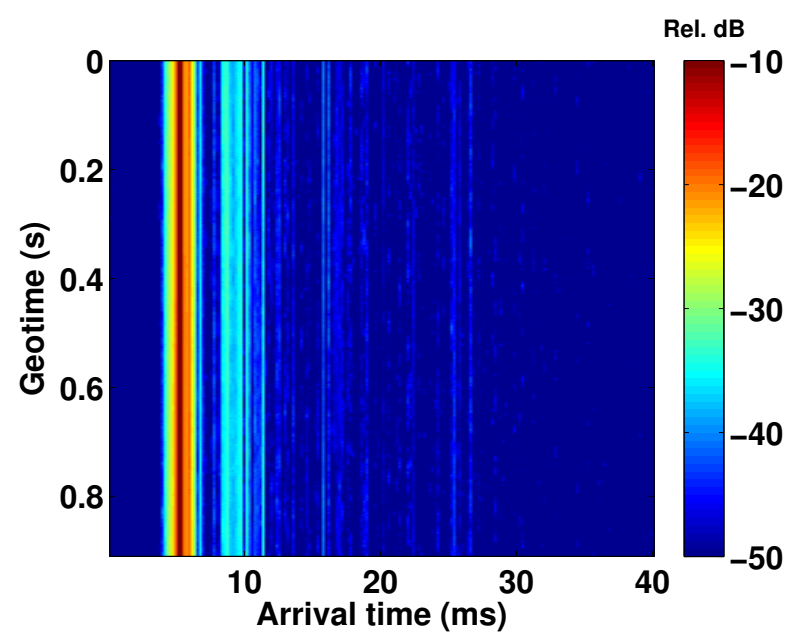

Figure 3: Estimated impulse responses at $30 \mathrm{kHz}$.

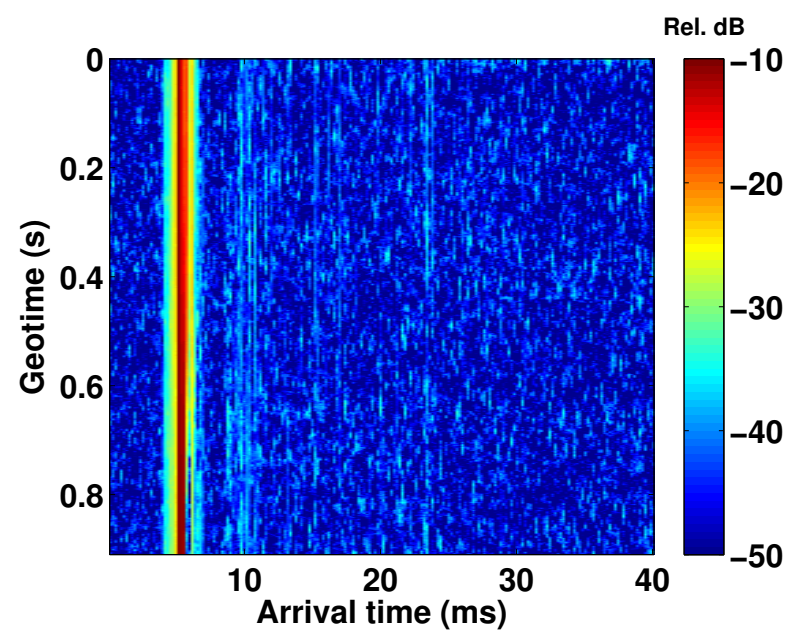

Figure 4: Estimated impulse responses at $95 \mathrm{kHz}$. 


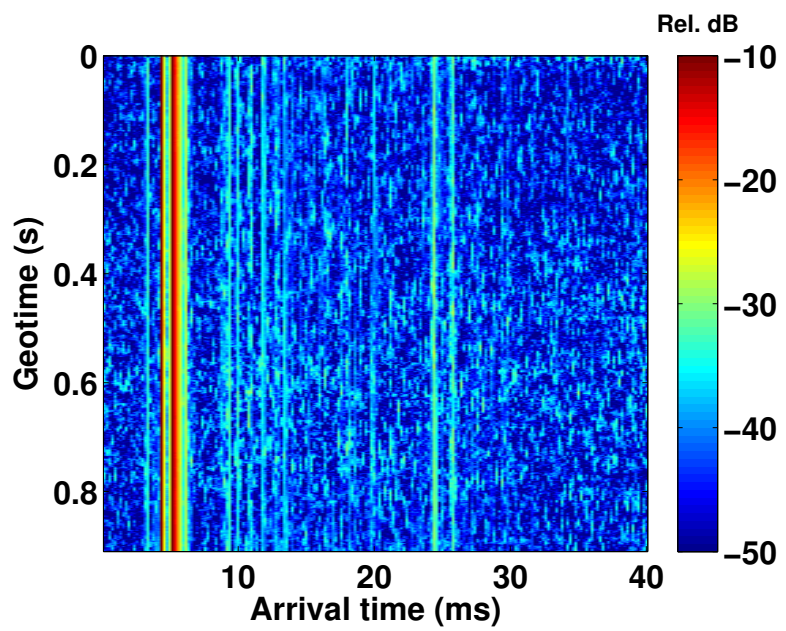

Figure 5: Estimated impulse responses at $180 \mathrm{kHz}$.

\subsection{CTIR}

We here adopt the coherent-to-incoherent intensity ratio (CTIR) metric [2] to characterize the self-interference channel within the one-second period.

The CTIR is defined as

$$
\eta(m)=\frac{\left|E_{n}[h[n ; m]]\right|^{2}}{E_{n}\left[\|h[n ; m]\|_{2}^{2}\right]},
$$

where $|\cdot|$ represents absolute value and $\|\cdot\|_{2}$ denotes $l_{2}$-norm. The expectation is applied over the packet duration in this work. The numerator in $\eta(m)$ is the coherently averaged intensity and the denominator is the incoherently averaged intensity for the path indexed by $m$ at time instant $n$.

We examine the impulse responses at three acoustic frequencies, 30, 95, and $180 \mathrm{kHz}$, and compute their corresponding CTIRs. The channel estimation method we used was threshold least squares. The channel impulse responses were integrated over a time duration of one second. The CTIR for each arrival was averaged over nine packets. As shown in Fig. 6, acoustic arrivals had much higher CTIR at $30 \mathrm{kHz}$ than those at 95 and $180 \mathrm{kHz}$. This was true, even for the strong arrivals at the arrival time of $5 \mathrm{~ms}$. The CTIRs at $5 \mathrm{~ms}$ decreased with increased frequency, even though the corresponding channel responses had similar strong returns at all three tested frequencies. This phenomenon is caused by the change of the channel phase. By analyzing the phase of these channels, we confirmed it that channel phase changed faster at higher frequencies. Also, later arrivals have lower CTIRs than that of the preceding ones. The same trend existed in CTIR plot at all three frequencies. This indicated that later arrivals were more scattered. The CTIR results confirmed that the arrivals at $30 \mathrm{kHz}$ were more stable, or more time-invariant.

The CTIR metric was aligned with the trend of IC gain with respect to the acoustic frequency. It is believed that higher IC gains can be achieved at lower frequencies since the self-interference channels are more stable.

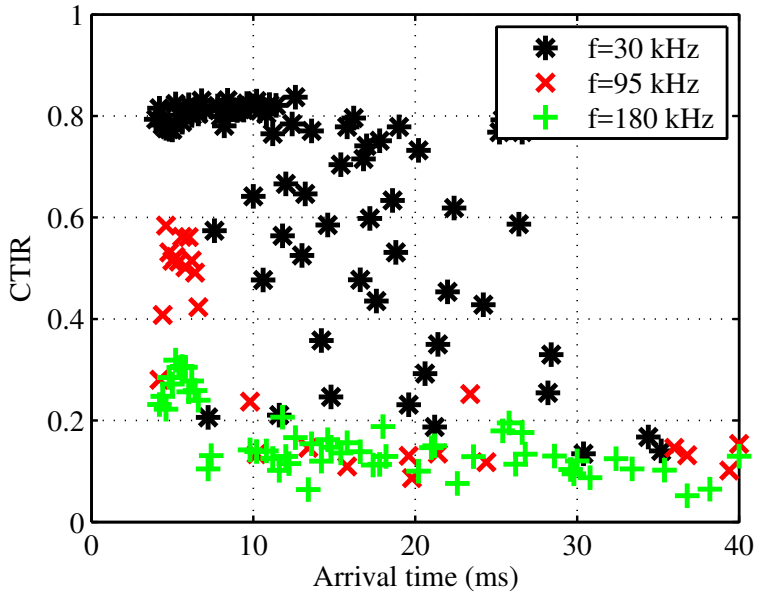

Figure 6: CTIR from the river experiment.

\subsection{CVR}

To quantify the channel dynamics, we split the acoustic channel into two parts, time-invariant and time-variant components. We assume the time-invariant part is stable over the entire observation window, which is twice the channel length. Thus the channel is denoted as

$$
\boldsymbol{h}^{t}=\boldsymbol{h}_{i}+\boldsymbol{h}_{v}^{t},
$$

where $\boldsymbol{h}^{t}$ is the channel impulse response vector at instant $t, \boldsymbol{h}_{i}$ is the time-invariant component and $\boldsymbol{h}_{v}^{t}$ is the time-varying component at instant $t$. In channel estimation, it is assumed that the channel impulse response is stationary over a period of time. If the channel experiences fast fluctuations, that $\boldsymbol{h}_{v}^{t}$ is present, it may bring difficulties to obtain true channel impulse responses.

Here, we assume the elements of the time-varying channel component, $\boldsymbol{h}_{v}^{t}$, follows Gaussian distributions with the mean of zero. If we take expectation of Eq. 7 during observation window $T$, we can obtain the time-invariant channel component as

$$
\boldsymbol{h}_{i}=E\left[\boldsymbol{h}^{t: t+T}\right] \text {. }
$$

where the expectation is applied over acquired channel samples.

Least square estimator is adopted for channel estimation. We can get multiple estimations, denoted as $\hat{\boldsymbol{h}}^{t}$, from observation window $T$. Using these estimations, we define the channel variation ratio (CVR) as

$$
\gamma=\frac{E\left[\left\|\hat{\boldsymbol{h}}^{t}\right\|_{2}^{2}\right]-\left\|\boldsymbol{h}_{i}\right\|_{2}^{2}}{E\left[\left\|\hat{\boldsymbol{h}}^{t}\right\|_{2}^{2}\right]}=1-\frac{\left\|\boldsymbol{h}_{i}\right\|_{2}^{2}}{E\left[\left\|\hat{\boldsymbol{h}}^{t}\right\|_{2}^{2}\right]} .
$$

where $E\left[\left\|\hat{\boldsymbol{h}}^{t}\right\|_{2}^{2}\right]$ is the intensity of the entire channel and the numerator denotes the intensity of the varying portion.

We calculated CVRs at all tested acoustic frequencies. The calculation was performed for $80 \mathrm{~ms}$, which was twice the channel length. To get enough estimations of $\boldsymbol{h}^{t}$, we re-estimated the channel each time the received signal sample got updated. The sampling rate in the channel estimation process was $5 \mathrm{kHz}$. Results are show in Fig. 7, where the CVR increases with the acoustic frequency. Of course, the increased CVR was the result of increased channel 
variation for high acoustic frequencies. The gradient of the CVR curve became smaller with the increase of frequency. This change of gradient was consistent with that in Fig. 2. This result, again, indicated the increase of CVR was the key cause of the IC gain decrease. Predictions can be made that we can achieve higher IC gains at even lower carrier frequencies.

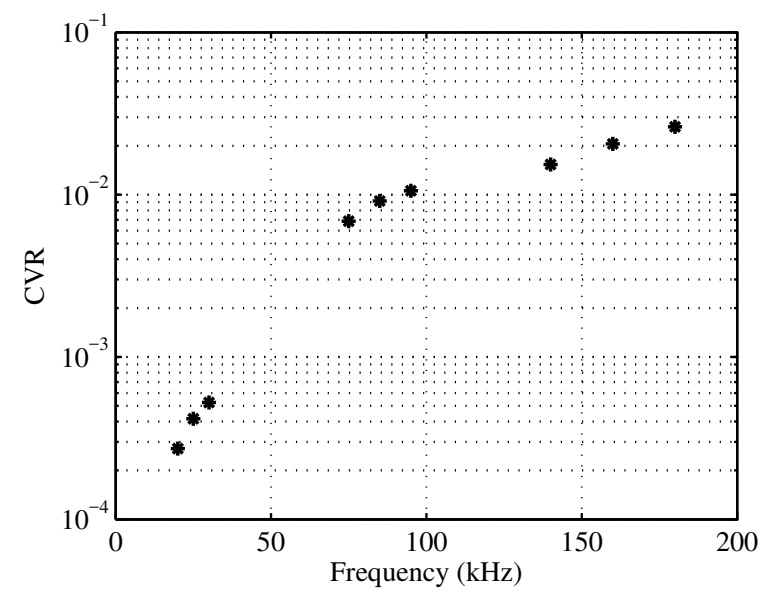

Figure 7: CVRs at nine acoustic frequencies.

To analyze the influence of channel fluctuations on IC gain, we simulated the IC performance for a time-varying channel. We generated the channel based on the two-component model in Eq. 7. We used the averaged impulse responses at $20 \mathrm{kHz}$ as the time-invariant component of the simulated channel. A total number of 41 paths were extracted from the measurement at $20 \mathrm{kHz}$. A time-varying component was generated based on Gaussian distribution. We assumed the later 10 paths, arrivals after $11 \mathrm{~ms}$, were time variant. To make each simulated $h_{v}^{t}$ naturalistic, a linear interpolation was applied between the generated Gaussian distributed numbers. By changing the intensity proportion of the two components, we got multiple simulated channels with different CVRs. The intensity proportion of the time-variant component ranged from 0.001 to 0.200 in the simulation. The interference to noise ratio (INR) in the experiment ranged from $72-94 \mathrm{~dB}$. We set the INR in our simulations to be $80 \mathrm{~dB}$.

Simulated IC gains are shown for different CVRs in Fig. 8, where the IC gains from the river experiment are shown for comparison. The two curves are almost parallel to each other. Both simulated and experimental IC gains decreased as CVR increased. The experimental IC gains were about $1.5 \mathrm{~dB}$ higher than that in the simulation for the same CVR. This difference between the two curves still needs further analysis. A possible reason may be a mismatch in the channel varying pattern. Plus, possible nonlinearity of the transmission/reception system is not considered in this work.

Based on both simulations and experimental analysis, we concluded that the channel variation was a key factor limiting the stability of the self-interference channel. This channel variation was the cause that degraded digital IC performance. This result suggests that a lower acoustic frequency is favorable to attain a higher IC gain in the digital IC.

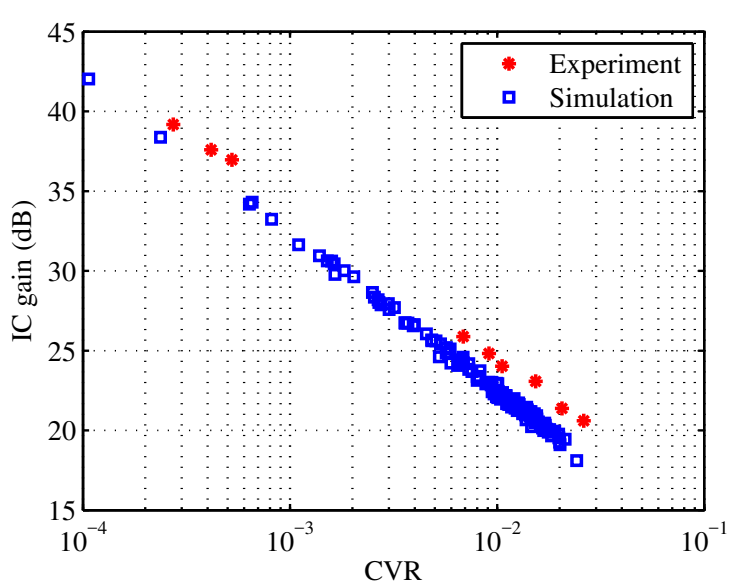

Figure 8: IC gain comparison.

\section{CONCLUSION}

We analyzed the relationship between IC gain and carrier frequency based on the field experiment conducted in a local river. Using digital cancellation methods, we reported that higher IC gains were obtained at lower frequencies. Furthermore, a channel variation metric, CVR, was proposed to quantify fluctuations of the selfinterference channel. It is well known that channel variation at higher frequencies is more intensive than that of lower frequencies. Naturally, the acoustic channel at higher frequencies had larger CVRs. We used computer simulations to show that higher CVRs led to performance degradation of digital cancellation in the selfinterference. It is believed that the channel variations contributed to the loss of the IC gain at the high acoustic frequencies.

\section{ACKNOWLEDGMENTS}

The efforts presented in this paper were partly supported by the NSF (CNS-1704097, CNS-1704076).

\section{REFERENCES}

[1] K. Bektas. 2004. Full-duplex Underwater Networking Using CDMA. Thesis.

[2] S. H. Huang, T. C. Yang, and C. Huang. 2013. Multipath correlations in underwater acoustic communication channels. The Journal of the Acoustical Society of America 133, 4 (2013), 2180-2190. https://doi.org/10.1121/1.4792151

[3] L. Li, A. Song, L. J. Cimini, X. Xia, and C. Shen. [n.d.]. Interference cancellation in in-band full-duplex underwater acoustic systems. In Oceans 2015 - MTS/IEEE Washington. 1-6. https://doi.org/10.23919/OCEANS.2015.7404411

[4] G. Qiao, S. Gan, S. Liu, L. Ma, and Z. Sun. 2018. Digital self-interference cancellation for asynchronous in-band full-duplex underwater acoustic communication. Sensors 18, 6 (2018), 1700

[5] G. Qiao, S. Liu, Z. Sun, and F. Zhou. [n.d.]. Full-duplex, multi-user and parameter reconfigurable underwater acoustic communication modem. In Oceans-San Diego, 2013. IEEE, 1-8.

[6] F. Qu, H. Yang, G. Yu, and L. Yang. 2017. In-band full-duplex communications for underwater acoustic networks. IEEE Network 31, 5 (2017), 59-65. https: //doi.org/10.1109/MNET.2017.1600267

[7] J. Tian, S. Yan, L. Xu, and J. Xi. [n.d.]. A time-reversal based digital cancelation scheme for in-band full-duplex underwater acoustic systems. In Oceans 2016 Shanghai. 1-4. https://doi.org/10.1109/OCEANSAP.2016.7485471

[8] G. G. Xie, J. H. Gibson, and K. Bektas. 2004. Evaluating the feasibility of establishing full-duplex underwater acoustic channels. Report. Naval postgraduate school Monterey CA dept of computer science.

[9] J. Zhang, X. Ma, G. Qiao, and C. Wang. [n.d.]. A full-duplex based protocol for underwater acoustic communication networks. In 2013 Oceans- San Diego. 1-6. https://doi.org/10.23919/OCEANS.2013.6741129 